\title{
Germanica
}

\section{Sabine Schos Schautiere}

Grit Dommes : Les animaux d'exposition de Sabine Scho

Grit Dommes: Sabine Scho's exhibited animals

\section{Grit Dommes}

\section{OpenEdition}

\section{Journals}

Édition électronique

URL : http://journals.openedition.org/germanica/6649

DOI : $10.4000 /$ germanica.6649

ISSN : 2107-0784

Éditeur

Université de Lille

\section{Édition imprimée}

Date de publication : 26 juin 2019

Pagination : 73-90

ISBN : 978-2-913857-42-1

ISSN : 0984-2632

\section{Référence électronique}

Grit Dommes, "Sabine Schos Schautiere", Germanica [Online], 64 I 2e trimestre 2019, Online erschienen am: 01 Januar 2021, abgerufen am 27 Februar 2021. URL: http://journals.openedition.org/germanica/ 6649 ; DOI: https://doi.org/10.4000/germanica.6649 


\section{Sabine Schos Schautiere}

Grit Dommes

Berlin

In allen Gedichtbänden von Sabine Scho finden sich Tiergedichte: die Pferdetexte ,-- auf Toro" und „Heidewind“ in Album (2001) ${ }^{1}$ und die Abteilung "Nachfolgende Tiere“ in farben (2008)2. Seit 2013 aber sind Tiere zu Schos Generalthema geworden: Der Band Tiere in Architektur versammelt Texte und Fotos zum Themenkreis Zoo und das Magazin The Origin of Senses, 2015, mit Zeichnungen von Andreas Töpfer und der Übersetzung von Ann Cotten, geht von Exponaten des Naturkundemuseums Berlin aus. Im Jahrbuch der Lyrik 2018 erschien Schos Gedicht ,gestrandeter pottwal“, das im gleichen Jahr auch in die Anthologie Aus Mangel an Beweisen von Michael Braun und Hans Thill aufgenommen wurde 3 . 2018 konnte Sabine Scho neben Christian Lehnert den Deutschen Preis für Nature Writing entgegennehmen, gestiftet vom Bundesamt für Naturschutz und Matthes \& Seitz, jenem Berliner Verlag, der in seiner Naturkunden-Reihe Naturund Kulturgeschichte zusammenbringt. Die Recherchen zu ihren Gedichten führen die Autorin in Zoologische Gärten, Nationalparks

1. - Sabine Scho, Album. Gedichte, Berlin, kookbooks, 2008 [zuerst: Hamburg/ Wien, Europa Verlag, 2001], S. 16, 25 ff.

2. - Sabine Scho, farben. Gedichte, Berlin, kookbooks, 2008, S. 52-68.

3. - Michael Braun, Hans Thill (Hrsg.), Aus Mangel an Beweisen. Deutsche Lyrik 2008-2018, Heidelberg, Wunderhorn, 2018, S. 70 f. Vgl. dazu Sabine Scho, „Did you see something? (Hast Du etwas gesehen?)“, Dritte Natur 2, 1/2019, S. 164-170, hier S. $169 \mathrm{f}$. Im Anschluss an diesen Text ist das Gedicht ebenfalls abgedruckt. 
und Naturschutzgebiete auf der ganzen Welt ${ }^{4}$. Und für eines ihrer aktuellen Projekte, die Bremer Netzwerkresidenz, sitzt Scho zurzeit an ihrem Schreibtisch auf einem „virtuellen Hochsitz“, beobachtet Webcams in Zoos und Nationalparks und kommentiert ihre Eindrücke in einem $B \log 5$.

Nicht nur das Thema verbindet die beiden letzten Publikationen, sondern auch die Bilder, die bei Scho fast immer eine Rolle spielen ${ }^{6}$. Auf die Texte folgt in Tiere in Architektur ein Bildteil, Fotos „mit Grünstich" (TA, 37), die von der Autorin und Matthias Holtmann stammen. Text- und Bildteile sind über Verweise wechselseitig aufein ander bezogen, ohne dass die Zuordnung restlos aufginge: Es gibt Texte ohne Bildbegleitung, aber auch umgekehrt Fotos, die für sich stehen. Das Magazin The Origin of Senses beginnt und endet mit den Zeichnungen von Andreas Töpfer, dem Chefgestalter des kookbooks-Verlags. Noch weniger als die Fotos sind diese Zeichnungen bloße Illustrationen. Sie unterstützen bisweilen das Verständnis, können aber den Zugang auch erschweren, weil sie ihrerseits ,gelesen werden wollen. Beide Bände weisen außerdem eine zusätzliche Kommentarebene auf: Den Gedichtband leitet ein gut verständlicher Essay ein, im Magazin begleiten ausführliche Anmerkungen, die ihrerseits wieder Fußnoten nach sich ziehen, die Gedichte. Der Essay ist eine ebenso knappe wie prägnante Kulturgeschichte des Zoos, die Anmerkungen geben Einblick in Schos Selbstverständnis als „Mängelwesen“7 , zu dem sich die studierte und belesene Autorin stets freimütig bekennt. Sie dokumentieren einige ihrer Arbeitsmaterialien, dabei stehen evolutionsbiologische Quellen und literarische Zitate gleichrangig nebeneinander und zeugen davon, dass die wichtigste Voraussetzung für ihr Schreiben die Recherche ist, sei es mit der Kamera vor Ort oder in den (virtuellen) Archiven des Wissens und der Kultur. Eine Grenzgängerin ist diese Autorin ohnehin, sie wechselt behände zwischen den Sprachen, Fachgebieten, Genres und Medien.

4. - Ende 2003 hielt sich Scho für ein dreimonatiges Residenzstipendium in der Villa Aurora in Los Angeles auf. Von 2006 bis 2013 lebte sie überwiegend in São Paulo, 2017 war sie als Artist in Residence in der Internationalen Naturschutzakademie auf der Insel Vilm bei Rügen, im Sommer 2018 als Grenzgänger-Stipendiatin in den Nationalparks Südafrikas und im Herbst 2018 mit einem Recherchestipendium der Kunststiftung NRW im Pantanal, dem größten Sumpfgebiet Brasiliens.

5. — https://www.netzresidenz.de/. Zugriff am 12.05.2019.

6. - Darüber informiert auch die biografische Notiz am Ende des Bandes: „Nahezu alle ihre Texte sind im Grenzbereich zu Fotografie und Bild angesiedelt.“ Sabine Scho, Tiere in Architektur. Texte und Fotos, Berlin, kookbooks, 2013, S. 126. Im Folgenden Sigle $T A$ und Seitenangaben im Text. Zitate aus Gedichten dieses Bandes, die ausführlicher analysiert werden, sind nicht im Einzelnen nachgewiesen.

7. — S. Scho, „Did you see something? ...“, a.a.O., S. 168. Vgl. auch TA, 39. 
Wie schon zu den Bildern kann man über die Anmerkungen sagen, dass sie den Zugang zu den Texten manchmal erleichtern, ,verlässliche Koordinaten und Sprecherinstanzen“ sucht die „durchblicksorientierte

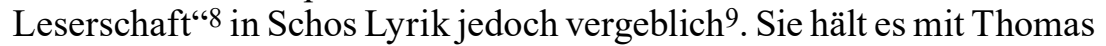
Kling, einem „,der Gründungsväter der heutigen Lyrik-Avantgarde in Deutschland"10, der in seinem Itinerar (1997) Gedichte als ,hochkomplexe (,vielzüngige', polylinguale) Sprachsysteme. Kommunikabel und inkommunikabel zugleich"11, definiert hat. Kling knüpfte in den achtziger Jahren an die Avantgardebewegungen seines Jahrhunderts an, an Hugo Balls Cabaret Voltaire, die Wiener Gruppe, Jandl und Mayröcker. In dieser Traditionslinie steht Schos Lyrik mit ihrer intensiven Arbeit am sprachlichen Material, die keineswegs auf die Muttersprache beschränkt bleibt ${ }^{12}$, und dem vorherrschenden Verfahren der Montage, das sich aus analogen und digitalen Textspeichern unterschiedlichster Art bedient, ohne „E und U auf getrennten Kontinenten anzusiedeln“13. Die Wirkung ihrer Texte ist, wie Metz es für die aktuelle Lyrik im Allgemeinen formuliert, ,zuerst auf das Denken ausgerichtet“ und will „erst von dort aus eine emotionale Bewegung erzeugen“14, ohne dass man diese Reihenfolge allzu dogmatisch auffassen sollte. Denn gelegentlich unterlaufen Schos Gedichte die Kategorisierung als ,schwere Kost' und überraschen mit Slang, Realsatire und Slapstick.

\section{„die stadt ist mein schauraum"15: Architektur für Tiere?}

Im Eingangsgedicht von Tiere in Architektur, „Das erste Tier“ (TA, 16 f.), betritt man den Berliner Zoologischen Garten vom Tiergarten

8. - Sabine Scho, „Avantgarden in der deutschsprachigen Dichtung um 2016. Ein Einblick“, https://www.goethe.de/ins/cz/de/kul/the/pro/21025645.html (Zugriff am 11.01.2019).

9. - Eine überzeugende literaturtheoretische Einordnung dieser Anmerkungen findet man in Mirjam Springer, "'Mit dem Rücken zum Publikum”? Sabine Scho: Album (2001) und Sabine Scho/Andreas Töpfer: The Origin of Senses (2015)“, in: Carlo Brune/Ines Theilen (Hrsg.), ,Wellenritt in riffreicher Zone" - Gegenwartslyrik im Deutschunterricht, Trier, Wissenschaftlicher Verlag, 2019 [im Druck].

10. - S. Scho, ,Avantgarden...“, a.a.O.

11. - Thomas Kling, Itinerar, Frankfurt a.M., Suhrkamp, 1997, S. 55. - Zur „Abweichungspoetik“ moderner Lyrik vgl. die prägnante Zusammenfassung in Leonhard Herrmann, Silke Horstkotte, Gegenwartsliteratur. Eine Einführung, Stuttgart, Metzler, 2016, S. 178.

12. - Zur „Multilingualität der Gegenwartslyrik“vgl. Christian Metz, Poetisch denken. Die Lyrik der Gegenwart, Frankfurt a.M., S. Fischer, 2018, S. 48.

13. - So formuliert es Kling für die von ihm ausgewählte Lyrik seit 1989: Sprachspeicher. 200 Gedichte auf deutsch vom achten bis zum zwanzigsten Jahrhundert eingelagert und moderiert von Thomas Kling, Köln, Dumont, 2001, S. 310.

14. - C. Metz, Poetisch denken..., a.a.O., S. 47.

15. - TA, 64. Mit dem Gedicht „são paulo stauraum“ nimmt Scho Bezug auf 
aus wie Walter Benjamin in der Berliner Kindheit um neunzehnhundert. „Tiergartenportal“"? Um die Wende zum vergangenen Jahrhundert hatte der Zoo tatsächlich noch drei Eingänge, heute gibt es keinen Zugang vom Tiergarten mehr. Der Wirklichkeitsabgleich sorgt für weitere Irritationen: Kamele findet man in der Nähe des Löwenportals, ihr Gehege ist tatsächlich von einem „wasserlosen Graben“ und einer Betoneinfassung umgeben, allerdings nicht kreisförmig. Manche Bäume im Zoologischen Garten tragen Namensschilder, aber bei den Kamelen steht keiner davon. Und von den drei Kamel-Fotos $(T A, 67)$ ist nur das untere mit den beiden Dromedaren im Berliner Zoo entstanden. Von Anfang an steht also fest, dass der Zoo in Tiere in Architektur beides ist: realer Ort und Textraum. Benjamins Erinnerungsbuch begeistert sich vor allem für den „Zwinger des Fischotters“, vor dem das Kind „endlos“ auf den „Insassen“ wartet, der sich jedoch „,nur für einen

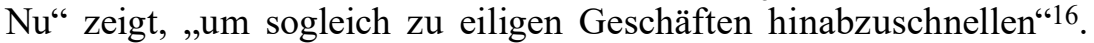
Schos Zoogängerin dagegen wird auf ihren Touren nicht nur „bald fußmüde“, sondern ist auch genervt von der Ignoranz der „Bewohner“: „hatte es über, nur immer zu schauen und kein einziges Mal angeblickt zu werden“. Eine rühmliche Ausnahme macht das Kamel: „Das erste Tier, das kam, wann ich wollte". Doch auch das Kamel kommt nicht aus Interesse oder gar Sympathie für die Betrachterin, es wird mit Eicheln bestochen - „Seltsame Früchte, die auf seinem Planeten nicht wuchsen." Zwar ist der Zoo ein Ort der Globalisierung, aber doch einer, der den je eigenen Standpunkt deutlich markiert. Der Text verkehrt gleich im ersten Abschnitt die Perspektive, das orientalische Tier wird zum Vertrauten und „Trauerbuche Europa“ sowie die deutschen Eicheln rücken exotisch auf Abstand; so tritt ein zentrales Thema des Gedichtbandes hervor: Es geht um den Blick und seine Richtung. Bereits der Titel Tiere in Architektur kündet davon: Der Mensch hat eine Kulturlandschaft um die Tiere herum gebaut, so hofft er, sie mit Hilfe eines ,definierten Parcours, kalkulierten Perspektiven, Sichtachsen, Aussichtsplattformen“ $(T A, 11)$ optimal präsentieren zu können. Auf dem Cover des Bandes, das sich zum Plakat entfalten lässt, hat Andreas Töpfer einen Käfig gezeichnet, der gleichzeitig ein Fotoapparat ist. Den hat auch die Besucherin im Eingangsgedicht dabei, sie ist nicht zur Zerstreuung in den Zoo gekommen, wie das „Altweltpublikum“ um sie herum, sondern auf „Fotosafari“. Ihr Auftrag ist ein quasi naturwissenschaftlicher, wie ihn das Zitat im zweiten Abschnitt formuliert: sehen, prüfen, exakt beschreiben, definieren. Von Aristoteles über Charles

Klings „Manhattan Mundraum“ aus morsch (1996).

16. - Walter Benjamin, Berliner Kindheit um neunzehnhundert. Mit einem Nachwort von Theodor W. Adorno, Frankfurt a.M., Suhrkamp, 1987, S. 44, 45. „Otter im Eimer" bezieht sich explizit auf Benjamins Fischotter-Kapitel (vgl. TA, 33). 
Darwin bis heute lautet so die Arbeitsbeschreibung des Zoologen. Und doch spekuliert das Ich neben dieser nüchternen Annäherung vor allem auf die Begegnung mit dem Kamel: „Mich freute, in seiner Umlaufbahn von Bedeutung zu sein, [...], denn nach ihm war ich allen anderen Tieren im Park gleichgültig." Die Sehnsucht nach Zuwendung sucht im Spiegel des Anderen auch das Eigene, so wie die „Gestaltung“ von Tiergärten „das Selbstverständnis einer Gesellschaft" spiegelt $(T A, 7)$. Zootiere als „Bewohner" von „Quartieren“ zu begreifen, ist deshalb weniger eine Frage der Achtung als vielmehr Ausdruck eines Narzissmus, der in der Postmoderne „Konjunktur“" hat ${ }^{17}$. In Antoine de Saint-Exupérys Le Petit Prince (1943) wird die Begegnung von Mensch und Tier so geschildert, wie der Mensch sie gerne hätte: Der Fuchs, dem ein bisschen langweilig ist, bittet den kleinen Prinzen: „S'il te plaît ... apprivoise-moi!" 18 . Und der kleine Prinz kommt von da an jeden Tag geduldig wieder, bis sie einander vertraut sind und gegenseitig ihre Einzigartigkeit erkennen. Schos Zoobesucherin erkennt auf der ,Spiegelfläche‘ ein Kamel - ein Schelm, wer Böses dabei denkt. Noch dazu kommt das Kamel wegen der Eicheln, die anderen Tiere bleiben ganz weg: ,Die Wege waren mir geläufig, die Tierhäuser bekannt, allein ihre Bewohner würdigten mich keines Blicks." Die Frage, wer eigentlich wen anschaut, und wenn ja, warum, wer ein- und wer ausgesperrt ist, zielt ins Zentrum der Zoothematik. „Sein Blick ist vom Vorübergehn der Stäbe / so müd geworden, daß er nichts mehr hält. / Ihm ist, als ob es tausend Stäbe gäbe / und hinter tausend Stäben keine Welt"19, heißt es in Rilkes Gedicht „Der Panther“ (1903) voller Empathie für das Energiebündel hinter Gittern. Die Perspektive dieses Textes führt den Leser durch den „Vorhang der Pupille“20 bis ins Herz des Tieres. Das sprechende Ich in Schos Gedicht wäre schon froh, wenn es wenigstens die Gehege betreten könnte, ,doch hatten die Fassaden weder Tür noch Tor. Man empfing nicht. Einlass hatte nur ein beflissenes Personal über verborgene Dienstbotenwege.“ Das Zoogelände, das ein Fenster zur Welt sein soll, erscheint als ,tierischer Irrgarten“, dessen Bewohner sich „erfolgreich“ hinter „Schutzwällen“ verbergen. Kulturgeschichtlich dokumentiert der Text den aktuellen Trend der Zoo-Architektur, „den

17. - Almut Barbara Renger, „Vorwort“, in: Dies. (Hrsg.), Mythos Narziß. Texte von Ovid bis Jacques Lacan, Leipzig, Reclam, 1999, S. 14-20, hier S. 14.

18. - Antoine de Saint-Exupéry, Le Petit Prince, Paris, Gallimard, 1990, S. 69. - Die wiederholt eingesetzte Planetenmetapher unterstreicht den Bezug zu SaintExupérys Erzählung.

19. - Rainer Maria Rilke, Werke in drei Bänden, Bd. 1, Gedicht-Zyklen, Frankfurt a.M./Leipzig, Insel Verlag, 1991, S. 261. - Vgl. die Gitter-Fotos unter der Überschrift „Stäbe gäbe“ in $T A, 114 \mathrm{f}$.

20. — R. M. Rilke, Werke..., a.a.O. 
Versuch, auf Augenhöhe ein Mit-Tier zu sein“21, und konstatiert ihn als gescheitert. Es ist der Mensch, der den Tieren enge Käfige oder luxuriöse Suiten zuweist, insofern klärt diese Architektur vor allem über ihn selbst auf. Benjamin begründet die Anziehungskraft, die der Zoologische Garten und besonders „die abgestorbenste Region des Gartens" auf ihn ausübte, mit der ,prophetischen Gabe" solcher Orte: „Verlassene sind es meist, [...] wo kein Mensch sich jemals aufhält. An solchen Orten scheint es, als sei alles, was eigentlich uns bevorsteht, ein Vergangenes." 22 Wie Benjamin der Fischotter zur Metapher des unbehausten Menschen wird, so interessieren Scho die Zoos als „Irrgärten“, aufgegebene Gehege oder verschlossene Orte, weil sie genau das wissen will: was uns Menschen noch bevorsteht. „Das erste Tier“ ist die Poetologie der Scho'schen Zoolyrik, der „Wiederkäuer“ „mit seinen geteilten Lippen" das selbstironische Alter Ego der Lyrikerin. Wenn gleich das erste Gedicht gesteht, dass es keinen Zugang zu dem eröffnen kann, was eigentlich festgehalten werden soll („Fotosafari“), dann darf der Leser weder mit Vogelperspektive noch mit Goldenem Schnitt rechnen. Postkartenmotive haben ihren Platz auf den Ständern, ,gleich hinter dem Tiergartenportal“. In der Ankündigung zu ihrem Seminar am Deutschen Literaturinstitut in Leipzig fragt Scho: „Was ist das überhaupt, ein dichterisches Motiv, und wie lässt es sich einfangen? Wie von etwas so nicht dichten, dass es gemeint ist?" 23

Wie Fotos und Spiegelbild, Menschenbild und Tierbild zusammenhängen, klärt der Text „Große Ohren, kleine Ohren“: „Sich auf Fotos nicht wiederzuerkennen, liegt am Selbstbild und am Verfall und daran, dass man sich sonst nur spiegelverkehrt im Spiegel sieht. Richtig? Richtig! Wer lebt schon gerne im Spiegelkabinett als Vampir?" $(T A, 38)$ Der Mensch als Blutsauger? Scho betreibt gewiss keine Greenpeace-Agitation ${ }^{24}$, lässt sogar kleine Spitzen gegen die „Artenschutzaktivisten“ $(T A, 30)$ nicht aus, aber ihre Texte haben eine Haltung, auch in Tierrechtfragen: Sie zeigt den begehrlichen menschlichen Blick aufs Tier, einen Blick voller Eitelkeit, Neid und Gier: „Diese Fellchen kann kein Weber wirken, Haar um Haar vertäute Garne, die sich zippergleich verzahnen. Kein zweiter Pelz so dicht und heute kaum mehr zu bezahlen", werden Bewunderung und Begehren im Rückgriff auf Else Lasker-Schülers „Tibetteppich“ kurzgeschlossen. „Sich schälen

21. - Zit. nach: Stefan Hochgesand, „Aus der Froschperspektive. Sabine Scho erkundet in Tiere in Architektur mittels Prosa und Fotos, warum wir skurrile Tier-Orte schaffen“, Tip 2/2014, S. 60.

22. - W. Benjamin, Berliner Kindheit..., a.a.O., S. 43.

23. - DLL, Vorlesungsverzeichnis 2018/19, S. 9.

24. - Vgl. S. Hochgesand, „Aus der Froschperspektive...“, a.a.O. 
lassen für Nietengürtel und Taschen", ist das Los der Echsen und natürlich fällt jedem zu Raubkatzen gleich das „kostbare Fell“ ein ${ }^{25}$. Im Blog der Bremer Netzwerkresidenz schreibt Scho begeistert über das farbenfrohe Gefieder von Perlhühnern, das Vivienne Westwood nicht besser hätte entwerfen können. Der Name der Modedesignerin ist verlinkt zu einem Artikel auf „Fashionnetwork“, in dem man erfährt, dass Westwood sich für die Erhaltung des Regenwaldes und die Cool Earth-Bewegung längst mehr engagiert als für ihre Kollektionen. Der Schulterschluss mit der veganen Punkikone mag chic sein, aber Scho weiß genau, wo sie hinwill:

Die Gier nach ihren Häuten, Pelzen, Zähnen und Federn finde ich schon verständlich, aber in der Oper rennt man ja auch nicht auf die Bühne und reißt den Sänger*innen die Kostüme vom Leib. Das Naturspektakel lässt sich nun mal wirklich besser erleben, wenn man begreift, dass ein Leopardenfell niemand dringender braucht als der Leopard selbst für seine Aufführung. Der schlüpft eben nach der Vorstellung nicht wieder in seine Jeans. Wäsche zum Wechseln fehlt ihm komplett ${ }^{26}$.

„Katzenhäuser vor der Kirche“ endet mit einer provokanten Frage an alle Katzenliebhaber, die es in der intellektuellen Leserschaft gewiss reichlich gibt: „Ein kleines Raubtier, für das man Futter in Dosen erfand, doch den Dosenöffner allein für sich, weil alles, was man alimentiert, bereitwillig Güte und Gnade annimmt?" (TA, 41) Immer aufs Neue muss der Mensch die Überlegenheit über das Tier behaupten, jedes Mittel scheint dazu recht. Das eigene Fremde wird gerne veräußert, „wie die Tiere“ ist ein geläufiger Kommentar zu Schamlosigkeiten aller Art. „Gehege im Aufbau“ schlägt eine andere Lesart dieser Floskel vor: „Was vielleicht hieße, wie scheue, behutsame Wesen.“ $(T A, 57)$

Im zweiten Text ${ }^{27}$ des Bandes spiegeln der alte und der neue Zoo die Soziologie der Filmstadt Los Angeles. Auch dieses Gedicht ist eher ein Suchbild fast ohne Tiere, wenn man von den metaphorischen 28 einmal absieht: Es handelt vom „Old Los Angeles Zoo“, der, als die Tiere 1965

25. - TA, 32 („Otter im Eimer“), TA, 37 („Echse“), TA, 54 („Großkatzen in Vegas“).

26. - https://www.netzresidenz.de/: „Natur putzt sich raus“, Blogeintrag vom 26.11.2018. Zugriff am 12.05.2019.

27. — „Aus aufgegebenen Gehegen. Old Los Angeles Zoo“ (TA, 19 ff.).

28. - Der zitierte „dog in me“, einer, der sich „zum Affen“ macht, ,, a mockingbird sings“, „a lost sheep“ und „das Brummen eines aufgebundenen Bären“. Norma Desmonds „monkey“ aus Sunset Boulevard ist selbst im Film schon tot und der „Mustang" einfach ein rotes Auto. Nur der „big bad wolf“"könnte noch Furcht verbreiten, aber auch er ist eher eine Märchenfigur, wie das ganze Thema für Märchenmotive geradezu prädestiniert ist. 
an den nordöstlichen Rand des Griffith Park in modernere Gehege umgesiedelt wurden, als Picnic Area erhalten blieb - als Ort für Erinnerungen an vergangene Zoobesuche mit der Familie und als Denkmal des Fortschritts in der Zoologie, wie es ein Schild verkündet, das man im Fototeil des Bandes lesen kann ${ }^{29}$. Dem Text wird der verlassene Ort mit eingebauten Picknickmöbeln - „Bärengelass mit fest installiertem Grill“, ,an Bänken und Tischen ist nicht zu rücken“ - zum „Raum für Projektionen“. Die Fotos dokumentieren eine Gefängnisarchitektur, die Obdachlosen als Notunterkunft dient, während man anderswo in derselben Stadt „,voll verglast“ wohnt, mit Sicht „auf den Pool“. Die Inschriften auf den Wänden ,verlassener Höhlen“" weisen den Ort als ein modernes Lascaux aus, an dem die Filmmetropole ihr anderes Gesicht zeigt, wie in Billy Wilders Sunset Boulevard (USA, 1949) „die Vergessenen, die von verblichenem Ruhm träumen, mit den Erfolglosen, die skrupellos für ihren Aufstieg kämpfen“"30, zusammentreffen: L.A. kann auch für „Lost Animals“ stehen oder das Gegenteil von Eden.

„Die Urkulisse jeder Menagerie“, schreibt Scho im Einleitungsessay, bleibt „der eingehegte Garten, der Hortus conclusus, als Rückzugsort, Paradies- und Lustgärtlein“ " $(T A, 14)$. Das Paradies ist verloren, das wissen Schos Gedichte genau. In „Asunción. Der unbetretbareZoo“ wird die Welt zu einer einzigen No-go-Area, in der nur noch ,in den kleinsten Ecken, den Blicken entzogenen Winkeln“ $(T A, 48)$ Überleben möglich scheint. Und doch gibt es Glücksmomente im ,falschen Leben'. Unter den Fotos fällt beim Blättern sofort das Otterbild $(T A, 82)$ auf, das, ohne Grünstich und mit ansehnlicherem Eimer, durchaus das Zeug zum Postkartenmotiv hätte. Im dazugehörigen Gedicht heißt es doppelzüngig: „Noah hat uns so ausgebootet, wir haben ab jetzt nur uns", und ein paar Zeilen weiter „schöner ist es doch, zu zweit zu verreisen und gemeinsam irgendwo zu stranden“ (TA, 32, 33). Da gibt sich das Gedicht, dessen Titel man wörtlich oder resignativ lesen kann, ganz kurz als Liebeslyrik zu erkennen, hat es doch auch schon Lasker-Schülers Hymne auf das Verwoben-Sein zweier Seelen zitiert, bevor es zurückkehrt zum Bewusstsein der Utopie: „Sich an irgendetwas halten, das bleibt ein Traum von zwei Ottern im Eimer" (TA, 33). Poetologisch knüpft der Text mit dem seit Klopstock bedeutungsschweren Motiv des Eimers an Thomas Klings Gedicht „di

29. — „Although these historic enclosures are no longer appropriate for housing animals, they can be home to memories of family visits to the Griffith Park Zoo, as well as an opportunity to better understand developments in the zoological sciences.“ $(T A, 70)$.

30. - Dieter Krusche unter Mitarbeit von Jürgen Labenski, Reclams Filmführer, 9. neubearb. und erw. Aufl., Stuttgart, Reclam j., 1993, S. 541. 
weite sucht"31 von 1991 an, in dem ebenfalls das empfindsame Potential moderner Lyrik ausgelotet wird:

di weite sucht

senti!,
das nach wi vor heftigste:
den sprachn das sentimentale
abknöpfn, als wär da nicht schon so gut
wi alles im eimer; bausch der im
hohn bogn in ein op-behältnis
fliegt;
$\quad$ ein nachtzug, draußn, der

In „Das Flamingo“ (TA, 22 ff.) geht es nicht um einen Zoo, hier ist das berühmte Hotel in Las Vegas der Schauplatz, das sich mit echten Flamingos schmückt. Die Besonderheiten dieser bonbonfarbenen Vögel werden erwähnt: Sie singen nicht, „fressen kopfunter“, „können die Farbe verlieren“ und beim Vorwärtsschreiten ,knicken die Knie nach hinten“. Eigentlich aber fingiert der Text einen Dialog zwischen zwei ganz anderen ,schrägen Vögeln': Benjamin ,Bugsy' Siegelbaum (19061947) und Meyer Lansky (1902-1983), Mitglieder der Kosher Nostra, einer US-amerikanischen, jüdisch dominierten Verbrecherorganisation nach dem Vorbild der italienischen Mafia. Siegelbaum war 1946 am Bau des Luxushotels mit Kasino und Flamingos beteiligt. Der Mobster wurde erschossen, als sich herausstellte, dass er Millionen US-Dollars auf einem Schweizer Nummernkonto beiseitegeschafft hatte, die für den Hotelbau gedacht waren. In Schos Dialog warnt Lansky Siegelbaum, er solle den Hotelbau aufgeben und stattdessen untertauchen, doch dieser zeigt sich siegessicher: „Meyer, ich bin am Ball, und wenn ich es in der Wüste schaffe, schaff ich es überall.“ Das liest sich im Vergleich $\mathrm{zu}$ den meisten anderen Texten leicht und erinnert im Ton an „Noah und seine Familie besteigen die Arche“, in dem Noah die Reihe der Tierpaare, die seine Arche besteigen, jovial dirigiert und kommentiert ${ }^{32}$. Sprachlich entfaltet Scho in „Das Flamingo“ die ganze Vielfalt der Vogelmetaphorik, lässt jiddische Wörter einfließen und das Glücksspiel-Business durchblitzen. Die Halbwelt, das große Geld und ein Mord - schnell wird klar, dass die ,Wasservögel in der Wüste“ kein „Artenschutzprogramm“ sind.

Las Vegas verdankt seine Bedeutung der etwa 50 Kilometer südlich im Black Canyon gelegenen Talsperre des Colorado. Die für

31. - Thomas Kling, brennstabm. Gedichte, Frankfurt a.M., Suhrkamp, 2. Aufl. 1997, S. 168.

32. — Vgl. S. Scho, farben..., a.a.O., S. 63 f. 
den Bau angeheuerten Arbeiter, die im nahegelegenen Boulder City wohnten, kamen zum Glücksspiel nach Las Vegas und brachten der Stadt Wohlstand und Aufschwung. Der riesige Staudamm wurde zwischen 1931 und 1935 errichtet, allein das Betonieren dauerte zwei Jahre. Herbert C. Hoover, dem 31. Präsidenten der USA, sollte dieses renommierte Bauprojekt zur Wiederwahl verhelfen und deshalb seinen Namen tragen, versprach es doch mitten in der Weltwirtschaftskrise Tausenden Arbeit, wenn auch unter verschärften Bedingungen. „Dam $\operatorname{Dog}^{\prime \prime}(T A, 24 f$.$) erinnert an diese Arbeiter, ,harte Hunde', die bereit$ waren, zur Not den „Blutzoll“" zu zahlen ,für vier Dollar am Tag“ und ein besseres Amerika. Der Text nimmt die rührselige Erzählung von Little Niggy auf, einem „schwarzen Bastard“, der den Arbeitern am Staudamm zulief und zu ihrem Maskottchen wurde 33 . Die Legende berichtet, er habe die Arbeiter mit seiner Leichtfüßigkeit auf Laufstegen hoch über dem Fluss erstaunt ${ }^{34}$ und diese hätten bei ihren Vorgesetzten erreicht, dass der Hund jeden Tag ein eigenes Lunchpaket bekam, für das die Arbeiter sogar bezahlten, bis er an einem heißen Tag unter einem LKW Schatten suchte und überfahren wurde, als dieser anfuhr. Die Arbeiter trauerten und begruben den Hund in einem selbst ausgehobenen Felsengrab ${ }^{35}$. „Man hat ihn hier eingemauert“, sagt die Stimme des ehemaligen Arbeiters als Fremdenführer in Schos Gedicht, ,und wenn man mich fragt, sag ich, man hat ihn lebendig begraben, denn das Wehr muss ja halten, tatsächlich aber war er schon tot." Da schiebt sich die andere Geschichte in den Text hinein, auf die schon das Motto verweist: Theodor Storms letzte Erzählung Der Schimmelreiter von 1888. In ihr wird der Deichgraf Hauke Haien Zeuge, wie die Arbeiter beim Deichbau einen Hund ,verdämmen“ wollen: ,[S]oll Euer Deich sich halten, so muß was Lebiges hinein! [...] Ein Kind ist besser noch; wenn das nicht da ist, tut's wohl auch ein Hund!"36 Hauke rettet den Hund und nimmt ihn seiner Tochter als ,Spielkamerad“37 mit. Doch das dramatische Finale der Novelle, in dem die Familie des Deichgrafen und auch der dem Aberglauben entrissene Hund der Sturmflut zum Opfer fallen, lässt Zweifel an der Durchsetzungsmacht beharrlicher Aufklärung. Der Hoover Dam ließ die Arbeiter als Underdogs zurück, sie waren durch den legendären Bau nicht reich geworden: „hohlwan-

33. — Vgl. https://viviennemackie.wordpress.com/2010/03/02/hoover-dam-dog/ (Zugriff am 14.1.2019).

34 . - , , [...] he learned to race very happily and easily on catwalks swinging 700 feet above the river. The men were amazed as most animals cannot do this.“ (Ebd.).

35. - Vgl. ebd.

36. - Theodor Storm, Der Schimmelreiter. Novelle, mit einem Nachwort von Wolfgang Heybey, Stuttgart, Reclam, 1963, S. 107.

37. - Ebd., S. 108. 
gige Cowboys, Double-Uglies, Muckers ohne Jakobsleiter", bloß mit Fahrstühlen, die sie in die oberen Etagen der Wolkenkratzer bringen ${ }^{38}$.

Wie „das Gedicht immer schon von Überschneidungen gelebt hat" 39 , ist es durch seine komprimierte Form gut geeignet, Merkmale anderer Gattungen aufzunehmen, narrative Elemente etwa, Dialoge und Monologe. Tiere in Architektur überschreitet die Grenze zur Prosa viele Male und doch scheint der Begriff, Gedicht' diesen Texten angemessen, die zwar häufig den Vers verabschieden, aber nie die rhythmisierte Sprache, den Reim nicht verachten, eine mehrschichtige Bildlichkeit und ein Geflecht von Referenzen aufweisen und vor allem so „starke Merkmale sprachlicher Gestaltung erkennen lassen“ 40 , wie das eben nur Lyrik kann.

Auch thematisch meidet Schos Lyrik Begrenzungen: „Dam Dog“ ist kein Zoogedicht, ebenso wenig wie ,gibt es biber in tegel?“”, „Das Flamingo“, „Katzenhäuser vor der Kirche“, „Die Katzen von La Recoleta“ und „Die Taubenschläge von Thessaloniki“. Wie im Essay weitet sich der Blick aus auf andere tierische Behausungen innerhalb der Stadt. Und schon im Gedichtband von 2013 gibt es einen Sog ins Naturkundemuseum, „rupturwundemuseum“ $(T A, 50)$, dem sich Scho für ihre Intervention 2015 gründlich widmete.

\section{„nur mut, evolution!'441 - im Naturkundemuseum}

Das Berliner Naturkundemuseum, das weltweit $\mathrm{zu}$ den fünf größten seiner Art zählt, hatte Sabine Scho 2015 zu einer Intervention im Rahmen des noch laufenden Projekts „Kunst/Natur“ eingeladen, dessen Zielsetzung es ist, „einen Experimentalraum für die Wechselwirkungen von Kunst, Museumspraxis und Naturforschung" $\mathrm{zu}$ eröffnen ${ }^{42}$. Scho lieferte zwölf Gedichte zu Sinnesorganen von Tieren, die in der Ausstellung auf großen Bannern zu lesen oder

38. - Die Verunglimpfung als ,Double-Ugly“ ist eine perfide Verschärfung des englischen Ausdrucks für ,hässlich', ,abstoßend'; ,mucker' ist im amerikanischen Englisch ebenfalls ein Schimpfwort (,Schuft'), im Deutschen bezeichnet „Mucker“ Scheinheilige und Heuchler (vgl. Lutz Röhrich, Lexikon der sprichwörtlichen Redensarten, Bd. 3, Freiburg u.a., Herder, 1995, S. 1055), diese Bedeutung schwingt trotz der englischen Pluralbildung noch mit, weil es sich ja ausdrücklich um Menschen handelt, denen der Himmel verschlossen ist; Otis Elevator Company ist der weltweit größte Produzent für Aufzugsanlagen - unverzichtbar für die Hochhausarchitektur. Auch beim Dammbau kamen Aufzüge zum Einsatz.

39. - Th. Kling, Sprachspeicher..., a.a.O., S. 110.

40. - L. Herrmann, S. Horstkotte, Gegenwartsliteratur..., a.a.O., S. 179.

41. - Sabine Scho/Andreas Töpfer, The Origin of Senses. An Intervention, Berlin, Naturkundemuseum, 2015, S. 19. Im Folgenden Sigle $O S$ und Seitenangaben im Text. 42. - http://kunst.naturkundemuseum-berlin.de/kunst-natur-interventionen/ (Zugriff am 26.01.2019). 
als Audiofiles, via QR-Code abrufbar, zu hören waren. Auch die Übersetzungen ins Englische von Ann Cotten wurden so präsentiert. Und auf weiteren, gleich großen Bannern zeigte Andreas Töpfer seine Zeichnungen, die ebenfalls die Sinneswahrnehmungen veranschaulichen. Dauerhaft sind die Zeichnungen, Texte und Übersetzungen in dem zur Ausstellung erschienenen großformatigen, hellorangen Magazin The Origin of Senses dokumentiert. Der Titel referiert auf Charles Darwins Hauptwerk The Origin of Species, das 1859 die Grundlagen der Evolutionsbiologie legte. Mit seiner Theorie einer schrittweisen Entstehung der Arten entzog Darwin der Überzeugung vom Menschen als Krone der Schöpfung den Boden und machte sich die zeitgenössische Theologie zum Feind. Dass seine Forschungen im 21. Jahrhundert noch immer nicht in allen Köpfen angekommen sind, kann eigentlich nur ein Witz sein (,kennen sie den“, OS, 17) ${ }^{43}$. Scho/Töpfers Buch der Sinne jedenfalls ist getränkt mit evolutionsbiologischen Erkenntnissen, Schos Anmerkungen weisen auf aktuelle Forschungsergebnisse hin und Töpfers Zeichnungen variieren die Evolutionsreihen, deren Urbild The Evolution of Man im kollektiven Gedächtnis fest verankert ist ${ }^{44}$.

Jeder kennt die fünf Sinne, über die der Mensch verfügt. Scho und Töpfer aber beschäftigen sich darüber hinaus mit dem Gleichgewichtssinn, dem elektrischen Sinn, der Propriozeption, dem Schmerz- und dem Gruppensinn, der Viszerozeption und dem magnetischen Sinn, also mit insgesamt zwölf Sinnen, jedem sind eine Doppelseite mit Zeichnungen und ein Gedicht zugeordnet. Vor allem dank der Anmerkungen lernt man also erstmal eine Lektion in ,diversität" $(O S, 19)$, staunt angesichts der Möglichkeiten, die die Natur hervorgebracht hat (Elektrorezeptoren!, Parthenogenese!), und wird daran erinnert, dass sie beim Menschen, wenn überhaupt vorhanden, keineswegs immer das höchste Level erreicht haben. Mit dem menschlichen Traum vom Fliegen im Gedicht „archaeopteryx“ geht es $\operatorname{los}^{45}$. Der Besucher des Naturkundemuseums wird also bei seinen Erwartungen abgeholt, kann manches Bildungserlebnis verbuchen, wird dann aber auf ganz anderen Pfaden ausgesetzt: Was haben wir eigentlich davon zu wissen, dass ein Hammerhai am Kopf mit Elektrorezeptoren ausgestattet ist $(O S, 18)$ ? Kann man als Mensch in die Haut eines Rochens schlüpfen, Bewegungs-, Kraft- und Lagesinn

43. - Die Kreationisten, vor allem evangelikale Christen in den USA, lehnen Darwins Erkenntnisse bis heute strikt ab.

44. - Es wäre außerdem lohnend, den Begriff der Liste aus der konkreten Poesie mit Töpfers Zeichnungen zusammenzudenken. Vgl. C. Metz, Poetisch denken ..., a.a.O., S. $22 \mathrm{f}$.

45. — Vgl. M. Springer, „“Mit dem Rücken zum Publikum”? ...“, a.a.O. 
inklusive, wie Töpfer es in seinen Zeichnungen durchspielt $(O S, 8)$ ? Wollen wir das überhaupt? Beim Hummeressen bestimmt nicht: „wird gekocht bei lebendigem leibe / was fühlt ein hummer dabei / für gewöhnlich interessiert das / hungrige einen dreck" $(O S, 23)$. Die Frage ,wie fühlt sich das an“ $(O S, 26)$ zieht sich leitmotivisch durch den Zyklus. Sie ist beides, Ausdruck einer wissenschaftlichen Neugier und Einforderung von Empathie. Unvermittelt befördert sie einen ins Grenzgebiet der Naturwissenschaften, zu der ,nichtendenwollenden Debatte um das Qualiaphänomen“ $(O S, 18)$ etwa, in der um verlässliche Angaben über das Bewusstsein gerungen wird. Und in die Zweifel über unsere Erkenntnismöglichkeiten mischt sich Ekel angesichts jenes Überlegenheitsmythos, der die Mensch-Tier-Beziehung noch immer beherrscht: Eben wird im Gedicht ,gorilla“ über die Unmöglichkeit berichtet, Augen zu präparieren, da schiebt sich in die Betrachtung des mächtigen Gorilla-Präparats mit Glasaugen eine Gewaltphantasie: „hauen sie diesem gorilla / ein veilchen, das bleibt sicher unbemerkt in seinem schwarzvioletten gesicht" $(O S, 17)$. Im 19. Jahrhundert wurde nicht nur der Gorilla entdeckt, sondern auch die These formuliert, der Mensch stamme vom Affen ab. Vor allem gegen den größten Vertreter der Menschenaffen richtete sich die Aggression, die diese Erkenntnis hervorrief. Davon leben zahlreiche Darwin-Cartoons ebenso wie der Film King Kong von 1932, in dem Mensch und tierisches Ungeheuer um ,die weiße Frau' konkurrieren. Die im Gedicht zitierte Redewendung „ein / auge werfen“ spiegelt nicht den Sehvorgang, „wo doch umgekehrt / zurückgeworfen wird, licht in / wellen/teilchen" $(O S, 17)$, wohl aber den typisch menschlichen Besitzanspruch. Alle vier Gorillaunterarten sind inzwischen durch das Ebola-Virus, Waldrodungen und Wilderei vom Aussterben bedroht. „Menschenaffenfleisch gilt [...] in wohlhabenden und urbaneren Gesellschaftskreisen Afrikas als Delikatesse."46 Noch brutaler als „gorilla“ verkehrt „schimpanse“ ein Gedicht aus William Carlos Williams Alltagslyrik, „This is just to say“47, in ein perfides Geständnis, das Sexualität zum Schauplatz von Berechnung, Rivalität und Unterwerfung macht. Da stellt sich im Geflecht der Bezüge zu der einen Redewendung (,seinem Affen Zucker geben'), die im Text erklärt ist, eine andere ein (,jemanden vernaschen') - und wiedermal ist das Opfer selbst schuld: ,er war so devot / verängstigt / und gierig“" $(O S, 22)$. Dass der Mensch den Struggle for Life als Krieg uminterpretiert hat, zeigt sich auch an den historischen Schneisen, die die Texte schlagen: Hatten die Briten ihren ,Naturhafen' Scapa Flow, so planten die Nationalsozialisten das Projekt ,Hummerschere', einen

46. - https://www.wwf.de/themen-projekte/bedrohte-tier-und-pflanzenarten/ gorillas/ (Zugriff am 27.03.2019).

47. - Abgedruckt in $O S, 22$. 
künstlichen Hafen auf Helgoland, mit dessen Hilfe sie Seeblockaden verhindern wollten $(O S, 23)$. Und in Südamerika, der Heimat des Großen Ameisenbären, fällt der Tropische Regenwald Stück für Stück den Brandrodungen zum Opfer $(O S, 26)$.

Zwei benachbarte Gedichte des Zyklus tragen im Titel jeweils zwei mit dem Kaufmanns-Und verbundene Tiernamen: ,frösche \& dinos" und ,anglerfisch \& chimäre" $(O S, 24 \text { f. })^{48}$. In beiden Fällen ist der zweite Teil der so entstandenen Paare zusätzlich mit Bedeutung aufgeladen: ,Dinos', der kindliche Kosename für die ausgestorbenen Riesenreptilien, lässt an Schleichtiere und Zeichentrickfilme denken. So hat der Mensch die mächtigen Urtiere nachträglich domestiziert. „Chimaera“ ist der Name eines „Unthieres“49 aus der griechischen Mythologie mit drei Köpfen (von Löwe, Ziege und Schlange) und dem Hinterleib eines Drachen, als ,Chimäre wird daher ein Trugbild oder Hirngespinst bezeichnet. Der Singular legt diese Bedeutung nahe, aber die Nachbarschaft zu ,anglerfisch“ ruft auch eine wenig bekannte Ordnung von Meeresfischen namens Chimären auf.

„frösche \& dinos“ simuliert eine Forschungsanordnung, bei der Tiere mit Musik konfrontiert werden. Mit den „boxen“, die „für kleine tiere“ andere sein müssen ,als für große tiere“, sind also hier gar nicht in erster Linie Behälter zum Transport von (Labor-)Tieren gemeint, sondern Lautsprecherboxen. Auch sonst findet sich viel akustisches Vokabular: „höhere frequenzen“, „,countertenor“, „vorspielen“, „akustisch“, „reingetrimmt" und immer wieder - insgesamt sieben Mal! - „hören“. Dass es um den Hörsinn geht, ist also evident. Von der biologischen Besonderheit des Gehörs bei Fröschen, die in den Anmerkungen ausführlich erläutert wird, gehen wahrnehmungspsychologische Überlegungen aus: „frösche hören / sowieso nur das, was sie / hören wollen", heißt es im Gedicht und die Anmerkung präzisiert „Geräusche von Fressfeinden und Artgenossen. Alle anderen Lautquellen nimmt ein Frosch erst gar nicht wahr." Und dann schließt sich noch ein einziger, hintersinniger Satz an: „Die Vermutung liegt nahe, dass das nicht nur Fröschen so geht." In der Tat ist auch die menschliche Wahrnehmung immer selektiv: Wir registrieren bestimmte Reize und blenden andere völlig aus, die Auswahl beruht dabei selten auf bewussten Entscheidungen. Solche Selektionsmechanismen reflektiert das Gedicht am Beispiel der Musik auch für die Kunst. Die Frage,

48. - Die in den folgenden Absätzen nicht belegten Zitate sind diesen beiden Seiten entnommen.

49. - Benjamin Hederich, Gründliches Mythologisches Lexikon, Reprographischer Nachdruck der Ausgabe Leipzig, Gleditsch, 1770, Darmstadt, Wissenschaftliche Buchgesellschaft, 1996, Sp. 702. 
welche Tonfrequenzen Tiere hören können, wechselt zu der ganz anders gelagerten Frage, welche Musik für Tiere ,interessant“ ist, die häufig gestellt wird, um den Nutzen von Kunst zu beweisen oder gar davon zu profitieren - so kurbelt etwa Beethoven angeblich die Milchproduktion von Kühen an. Meistens geht es in diesem Zusammenhang um die beruhigende, stressmindernde Wirkung von Musik. Kate Bushs Song „And dream of sheep" ist da allerdings nur scheinbar ein passendes Beispiel. Der Songtext schildert einen Überlebenskampf, in dessen Verlauf das Ich sich nach ,traumschafe[n]“"sehnt. Bushs Album Hounds of Love von 1985 war ihr erfolgreichstes, was wahrscheinlich auf die A-Seite mit dem Titelsong zurückzuführen ist: Von den hier versammelten fünf Songs wurden vier als Singles ausgekoppelt. Die Kritik aber lobt vor allem den künstlerischen Eigensinn der B-Seite, die mit „And dream of sheep“ beginnt. Das Gedicht treibt seine grotesken Überlegungen zum Musikgeschmack von Tieren auf die Spitze, indem es spekuliert, was wohl Dinosaurier mit einer ganz bestimmten Arie aus Henry Purcells barocker Semi-Oper King Arthur (1691) anfangen könnten. Die Arie aus der Frost-Szene des dritten Akts ist eigentlich für eine Bassstimme notiert, wurde aber im 20. Jahrhundert durch den Countertenor Klaus Nomi als „Cold Song“ berühmt und in der Folge auch von anderen bekannten Künstlern, etwa von Sting, interpretiert. Der Schlussvers lautet „Let me, let me, / Freeze again to death!“"50. Wie dieses todessüchtige Lied aus einer spezifischen Form der Barockoper, der keine lange Dauer beschert war, die aber dem Sprechtheater eine Zeitlang Asyl bot ${ }^{51}$, durch die Interpretation eines UndergroundKünstlers, dem es gelang, David Bowies Aufmerksamkeit zu erregen, in der Moderne zu neuem Leben erweckt wurde, ist ein Paradestück der Kunst-Evolution. Diese Wandlungsfähigkeit eines Kunstwerks kommt beinahe der von Fröschen gleich: Im Gegensatz zu den Dinosauriern, für deren Aussterben die Wissenschaft noch keine eindeutige Ursache benennen kann, sind Frösche extrem anpassungsfähig, überleben längere Perioden von Trockenheit und Frost.

Auch ,,anglerfisch \& chimäre“ nimmt Bezug auf ein Lied. Das vollzieht sich erstmal rein assoziativ: Anglerfische verfügen nicht über eine Schwimmblase, sondern bewegen sich eher laufend über den

50. — https://lyricstranslate.com/de/klaus-nomi-cold-song-lyrics.html (Zugriff am 01.04.2019).

51. - Eine Halb-Oper ist eine Mischung aus Oper und Sprechtheater, in der nur die Nebenfiguren singen, „während die Hauptfiguren sich ausschließlich in der gesprochenen Rede artikulieren", so dass im England nach der puritanischen Revolution „mit Hilfe der Musik das vom Verbot bedrohte Sprechtheater überwintern konnte.“ (Ulrich Schreiber, Die Kunst der Oper. Geschichte des Musiktheaters, Bd. 1: Von den Anfängen bis zur Französischen Revolution, Frankfurt a.M., Büchergilde Gutenberg, 1988, S. 173.) 
Meeresgrund. Ihre Nahrung locken sie mit Hilfe einer Angel an, deren Köder bei den Tiefseeanglerfischen lumineszierende Bakterien enthält, also leuchtet. Ein Fisch, der auf seinen Brustflossen steht und dabei einen Angelbogen vor sich hat, erinnert an Manneken Pis, jene Brunnenfigur aus dem 17. Jahrhundert, die zu Brüssels Wahrzeichen geworden ist und zahllose Verwandte auf privaten und öffentlichen Brunnen in aller Welt hat. Nimmt man das Leuchtorgan dazu, das wie eine Laterne über dem Fisch schwebt, schiebt sich „Lili Marleen“ ins Bild:

\section{pinkelt der anglerfisch \\ mit den kleinen kiemen \\ dort bei der laterne \\ wie einst lili marleen \\ im stehen?}

Das erste Wort und der verkürzte Schlussvers dieses Abschnitts sind so ausgestellt unpoetisch, dass sie Komik erzeugen. Doch gleichzeitig klingt mit dem zitierten Refrain eine sentimentale Stimmung an, die den kalauerhaften Einstieg unterläuft. Im „Lied eines jungen Wachtpostens“ von Hans Leip, das unter dem Titel „Lili Marleen“ berühmt wurde, beleuchtet die Laterne zunächst das Glück eines Paares und dann dessen Trennung durch den Zapfenstreich, der den Soldaten in die Kaserne ruft. Nur angedeutet wird die viel radikalere, oft endgültige Trennung durch den Krieg: „Alle Abend brennt sie / Mich vergaß sie lang / Und sollte mir ein Leids geschehn / Wer wird bei der Laterne stehn // Mit dir / Lili Marleen?"52 Die Schlussstrophe imaginiert ein traumhaftes Auferstehungsszenario und lässt die Liebe über den Tod triumphieren. Die Laterne bleibt über das einzelne Schicksal hinaus das Dingsymbol dieses Versprechens. Kein Wunder, dass „Lili Marleen“, gesungen von Lale Andersen, 1939 zum „Soldatenschlager“" avancierte. Der Liedtext, in dem sich Heimweh und Todesangst mit der Hoffnung auf ein Wiedersehen mischen, taugte für Soldaten aller Kriegsparteien zur Identifikation. Von den Nazis wurde das Lied schließlich verboten. Schos Gedicht beginnt launig mit dem Fisch, der stehen kann, und endet beim sprechenden Ich, das sich anschickt, baden zu gehen, sich wie ein Fisch im Wasser fühlen möchte. Dazwischen verliert die kumpelhafte Sprecherinstanz ${ }^{53}$, die durch den Text führt, ihre Souveränität. Der Small Talk weicht eindringlichen Ermahnungen und Desillusionierungen: „schwarz angeln ist hier verboten / [...] schwärmen sie / nicht von der gruppe [...] / sie kämpfen allein [...]

52. - https://genius.com/Lale-andersen-lili-marleen-lyrics (Zugriff am 01.04.2019).

53. - Obwohl die Angesprochenen durchgehend gesiezt werden, herrscht ein salopper Ton: ,ich nehme sie nicht auf / den arm“, „darauf stoßen wir jetzt an“. 
mehr licht gibt es nicht / nein!“ Mit der Liedzeile „und steht sie noch davor" fallen die Kriegstraumata förmlich über die zoologischen Erläuterungen und Strandurlaubsimpressionen her, es entsteht eine Text-Chimäre, in der man sich beim Lesen leicht verheddert. Besonders am alliterationsreichen Ende des vierten Abschnitts, wo vom „gleich/ schritt gekühlter getränkter" die Rede ist, Drinks an der Strandbar von Wasserleichen überlagert werden. Innerhalb des Zyklus ist diesem Text der Gruppensinn zugeordnet, der die Ordnung in Fischschwärmen gewährleistet. Den Menschen fehlt dieser Sinn, ihr Gleichschritt läuft auf den Zusammenstoß hinaus, der sie, allem Kameradschaftskult zum Trotz, zu Einzelkämpfern macht. Das finale Baden-Gehen bezeichnet im übertragenen Sinn einen glanzlosen Untergang.

Die an ,anglerfisch \& chimäre“ gezeigte Sprechsituation ist typisch für viele Scho-Gedichte. Nicht selten kommt das sprechende Ich mit dem Gestus von Museums- oder Fremdenführern daher, die sich, mal mehr, mal weniger im Bilde ${ }^{54}$, an ein Kollektiv wenden: Wissen vermittelnd, um Aufmerksamkeit bemüht, immer mittendrin. Auktoriale Erzähler findet man in Schos Werk ebenso wenig wie die intime Gefühlsschau zwischen Ich und $\mathrm{Du}$, für die Lyrik gemeinhin als prädestiniert gilt. Schos Blick auf die Evolution entspringt einem brennenden Interesse an Aufklärung über sich selbst. „Das schien mir immer die eigentliche Lehre der Evolution zu sein, deren Teil wir ja sind“, sagte die Dichterin bei der Verleihung des Preises für Nature Writing, ,dieses Sich-selbst-erkennen-Können, gerade weil man verschieden ist in der Dazugehörigkeit." ${ }^{55}$

Das Naturkundemuseum selektiert und klassifiziert die Überreste toter Tiere und ausgestorbener Arten. Es lenkt den Blick des Betrachters und versorgt ihn mit Erklärungen wie die Schilder im Zoo. Geordnet liegen die Mineralien in den Vitrinen, in Reih und Glied stehen die Feuchtpräparate auf den Regalen, artgerecht arrangiert posieren die Vollpräparate in den Sälen, stumme Zeugen für die Behauptung, der Mensch habe die Welt im Griff. Für das gebrochene postmoderne Bewusstsein birgt dieses Konzept „die verheißung / auf heilung und pflege und krustenbildung“, als wären die Vitrinen gefüllt mit Verbandsmaterial (,mull“, TA, 50). Sabine Schos Gedichte stiften Unordnung, säen Zweifel. Sie sind Einlassungen und Einwände zugleich. Mit ihrer spezifischen Poetik des Widerstands halten sie, aus der Perspektive der Betroffenheit, die Wunden offen, und kennen doch auch Linderung: „wuischt a pflasta?“ (ebd.).

54. — „diesen alligator / dieses krokodil, ich frag' / besser den kurator (später)” $(O S, 20)$.

55. - Die Rede ist in der Zeitschrift Dritte Natur abgedruckt: S. Scho, „Did you see something? ...", a.a.O., S. 170. 
